\title{
Correlations Between Visual Acuity, Presenting Symptoms and Histopathological Findings in Enucleated Eyes with Uveal Melanoma
}

Elin Asplund

Karolinska Institute

Tony Pansell

Karolinska Institute

Rune Brautaset

Karolinska Institute

Maria Nilsson

Karolinska Institute

Gustav Stålhammar ( $\nabla$ gustav.stalhammar@ki.se )

Karolinska Institute

\section{Research Article}

Keywords: Uveal Melanoma, Choroidal Melanoma, Visual acuity, Symptoms, Retinal detachment, Histopathology, Vasculogenic mimicry

Posted Date: October 8th, 2021

DOI: https://doi.org/10.21203/rs.3.rs-952903/v1

License: (c) (1) This work is licensed under a Creative Commons Attribution 4.0 International License. Read Full License 


\section{Abstract}

Visual outcomes after treatment of uveal melanoma have been investigated repeatedly. In this paper, we evaluate the correlation between visual acuity (VA) prior to enucleation, presenting symptoms and findings upon histopathological examination of eyes and tumors. Sixty nine patients were included. Their mean best corrected visual acuity (BCVA) prior to enucleation was LogMAR 0.80 (SD 0.70). Patients that reported low VA or blurry vision did not have lower BCVA upon refraction $(p=0.34)$. Patients with low BCVA had tumors with greater apical tumor thickness $(p=0.027)$, basal diameter $(p=0.012)$ and stage $(p=0.014)$. The experience of a shadow in the visual field correlated with presence of vasculogenic mimicry in the tumor $(p<0.001)$. In multivariate Cox regressions with tumor thickness and diameter as covariates, tumor cell type and infiltration of the optic nerve head were associated with increased hazard for melanomarelated mortality (3.2 and 3.6, respectively). We conclude that patients that report low VA do not necessarily have worse BCVA. The latter do however have tumors with larger dimensions, at more advanced stage. A shadow in the VF correlates with presence of vasculogenic mimicry, which is an independent predictor for poor prognosis along with tumor infiltration of the optic disc and tumor cell type.

\section{Introduction}

Uveal melanoma is the most common primary intraocular malignancy, affecting more than 7000 individuals each year worldwide. ${ }^{1}$ Uveal melanomas most commonly arise in the choroid ( $90 \%$ of cases), followed by the ciliary body (6\%) and iris (4\%). ${ }^{2}$ Within 15 years from diagnosis, 32 to $45 \%$ of patients succumb to metastatic disease, with no survival difference after enucleation or eye-preserving plaque brachytherapy of eyes with medium-sized tumours. ${ }^{3-5}$

Several studies have reported short and long-term visual outcomes after plaque brachytherapy. These indicate that one-half of patients lose six or more lines of visual acuity (VA) three years after treatment. ${ }^{6}$ At 10 years, one-half to three in five patients have Snellen $<20 / 200$ and three quarters have abnormal visual field (VF) sensitivity (10-2 testing). ${ }^{7-10}$ Further, greater tumor thickness, shorter distance to the macula, subretinal fluid, worse baseline VA, non-Caucasian race, specific tumor shapes and a simultaneous diabetes diagnosis have been associated with increased risk for poor VA, with more than $99 \%$ of patients suffering from Snellen $<20 / 200$ if all factors are present. ${ }^{11}$

The most common presenting symptoms in uveal melanoma are blurred vision, a shadow in the VF, photopsia, floaters and metamorphopsia. ${ }^{12} \mathrm{~A}$ shadow in the VF, experienced by approximately 1 in 6 patients, correlates to an exudative retinal detachment and is an independent predictor of uveal melanoma-related mortality, regardless of other simultaneous symptoms, tumor size, location, local extent, or stage. ${ }^{13}$

Histologically, uveal melanomas are identified as dome-shaped, mushroom-shaped or lobulated masses of atypical melanocytes with varying degrees of pigmentation and vascularity. ${ }^{14-19}$ Typically, eyes that 
have been enucleated with uveal melanoma are examined by dedicated ophthalmic pathologists or by pathologists with ophthalmic pathology as a special interest. While many studies have examined histopathological and cytological findings in eyes with uveal melanoma that have undergone enucleation, we are not aware that anyone has connected the three: Histological findings, patients' reported symptoms and VA before removal of the eye. ${ }^{17,20-25}$

We therefore see an opportunity to examine the correlation between clinical, clinicopathological and histopathological factors in a retrospective cohort with long follow-up time.

\section{Results}

\section{Descriptive statistics}

Of the 68 patients, $42(62 \%)$ were men and $26(38 \%)$ were women. The mean age at diagnosis was 63 years (SD 12). The mean tumor thickness was $7.7 \mathrm{~mm}$ (SD 3.4) and the mean diameter $13.8 \mathrm{~mm}$ (SD 5.0 $\mathrm{mm}$ ). The mean BCVA of the tumor eye prior to enucleation was 0.80 (SD 0.70). Forty-nine patients (72\%) had high BCVA (LogMAR < 1.00$)$ whereas 19 patients $(28 \%)$ had low BCVA.

Fifty-three patients (78 \%) had deceased before the end of follow-up. Median follow-up for the 15 survivors was 16.6 years (SD 2.4, Table 1 ). 
Table 1

Characteristics of patients and tumors included in this study. SD, standard deviation. BCVA, best corrected visual acuity. AJCC American Joint Committee on Cancer.

\begin{tabular}{|c|c|}
\hline$n$ & 68 \\
\hline Mean age at diagnosis, years (SD) & $63(12)$ \\
\hline \multicolumn{2}{|l|}{ Sex, n (\%) } \\
\hline Female & $26(38)$ \\
\hline Male & $42(62)$ \\
\hline \multicolumn{2}{|l|}{ Tumor eye laterality, $n$ (\%) } \\
\hline Right & $35(51)$ \\
\hline Left & $33(49)$ \\
\hline Mean tumor thickness, mm (SD) & $7.7(3.4)$ \\
\hline Mean tumor diameter, mm (SD) & $13.8(5.0)$ \\
\hline BCVA, mean LogMar (SD) & $0.80(0.70)$ \\
\hline \multicolumn{2}{|l|}{ BCVA, classification } \\
\hline High (LogMar <1.00), n (\%) & $49(72 \%)$ \\
\hline Low (LogMar $\geq 1.00), \mathrm{n}(\%)$ & $19(28 \%)$ \\
\hline \multicolumn{2}{|l|}{ AJCC T-category, n (\%) } \\
\hline 1 & $11(16)$ \\
\hline 2 & $17(25)$ \\
\hline 3 & $22(32)$ \\
\hline 4 & $18(27)$ \\
\hline \multicolumn{2}{|l|}{ AJCC stage, n (\%) } \\
\hline I & $7(10)$ \\
\hline$\| \mathrm{A}$ & $20(29)$ \\
\hline IIB & $15(22)$ \\
\hline IIIA & $18(26)$ \\
\hline IIIB & $8(12)$ \\
\hline IIIC & 0 \\
\hline
\end{tabular}




\begin{tabular}{|ll|}
\hline$n$ & 68 \\
\hline IV & 0 \\
\hline Median follow-up, years (SD) & $16.6(2.4)$ \\
\hline
\end{tabular}

Upon histological examination, sixty-one eyes (90\%) had any degree of retinal detachment. Forty-four eyes $(65 \%)$ had a serous exudate. Five tumors $(7 \%)$ penetrated Bruch's membrane, $14(21 \%)$ infiltrated the ciliary body while $6(9 \%)$ and $3(4 \%)$ also infiltrated the anterior chamber angle and the iris, respectively (figure 1 ).

Nine tumors (13\%) infiltrated the optic nerve head, with all nine being limited to prelaminar invasion only and none extending beyond lamina cribrosa. Six tumors (8\%) had necrotic areas. The mean diameter of these necrotic areas was $3.6 \mathrm{~mm}$ (SD 2.5). Two patients (3\%) had extraocular tumor growth. The mean diameter of the extraocular tumor growth was $3.6 \mathrm{~mm}$ (SD 3.5). Nine tumors (13\%) were heavily pigmented (cell borders, cytoplasmic organelles and nuclei obscured), 22 (32\%) were composed of $>90 \%$ epithelioid cells, 35 (51\%) of mixed cell types and 11 (16\%) of $>90 \%$ spindle cells (figure 2$)$. The mean number of corneal endothelial cells per high power field $(\times 400)$ was 5.6 (SD 3.5, Table 2). 
Table 2

Histopathological findings. SD, standard deviation

\begin{tabular}{|ll|}
\hline Histopathological findings & \\
\hline Retinal detachment, $\mathrm{n}(\%)$ & $61(90)$ \\
\hline Serous exudate, $\mathrm{n}(\%)$ & $44(65)$ \\
\hline Bruch's membrane tumor penetration, $\mathrm{n}(\%)$ & $5(7)$ \\
\hline Optic nerve head infiltrated by tumor, $\mathrm{n}(\%)$ & $9(13)$ \\
\hline Ciliary body infiltrated by tumor, $\mathrm{n}(\%)$ & $14(21)$ \\
\hline Anterior chamber angle infiltrated by tumor, $\mathrm{n}(\%)$ & $6(9)$ \\
\hline Iris infiltrated by tumor, $\mathrm{n}(\%)$ & $3(4)$ \\
\hline Heavily pigmented tumor, $\mathrm{n}(\%)$ & $9(13)$ \\
\hline Tumor cell type, $\mathrm{n}(\%)$ & \\
\hline Epithelioid & $22(32)$ \\
\hline Mixed & $35(51)$ \\
\hline Spindle & $11(16)$ \\
\hline Corneal endothelial cells/hpf, mean (SD) & $5,6(3,5)$ \\
\hline Tumor necrosis, $\mathrm{n}(\%)$ & $6(8)$ \\
\hline Tumor necrosis, mean diameter (SD) & $3.6(2.6)$ \\
\hline Extraocular extension, $\mathrm{n}(\%)$ & $2(3)$ \\
\hline Extraocular extension, mean diameter (SD) & $3.6(3.5)$ \\
\hline
\end{tabular}

\section{Visual acuity versus patient age, tumor size, stage and histopathological findings}

Patients with low BCVA were not significantly older at diagnosis (64 years, SD 8.1 versus 61 years, SD 13.4, Mann Whitney $U \mathrm{p}=0.27)$ but had tumors with significantly greater apical tumor thickness $(9.2 \mathrm{~mm}$, SD 3.8 versus $7.1 \mathrm{~mm}, \mathrm{SD} 3.1, \mathrm{p}=0.027)$ and basal diameter $(16.4 \mathrm{~mm}, \mathrm{SD} 5.0$ versus $12.7 \mathrm{~mm}, \mathrm{SD} 4.7$, $p=0.012$ ). Similarly, they had significantly more advanced AJCC T category and stage (one-way ANOVA $p=0.009$ and 0.014 , respectively).

Contrarily, serous exudates (Pearson Chi-Square $p=0.77$ ), retinal detachments (Fisher's exact $p=0.31$ ), tumor infiltration of the optic nerve head $(p=1.0)$, extrascleral growth $(p=1.0)$, tumor penetration of Bruch's membrane $(p=0.62)$, tumor infiltration of the ciliary body $(p=0.74)$, tumor infiltration of the anterior chamber angle $(p=1.0)$, tumor infiltration of the iris $(p=1.0)$, heavy tumor pigmentation $(p=0.43)$, 
vasculogenic mimicry ( $p=0.14$ ), tumor cell type (one-way ANOVA $p=0.39$ ), tumor distance to the optic disc (Mann-Whitney $\cup p=0.12)$, tumor distance to the macula $(p=0.38)$ and the number of corneal endothelial cells/hpf $(p=0.76)$ were equally distributed between patients with high and low BCVA.

Patients with tumors that infiltrated the ciliary body had a mean BCVA of 0.78 (SD 0.72), versus 0.82 for patients without ciliary body infiltration (SD $0.69, p=0.66$ ). In all 5 cases where the tumor infiltrated the anterior chamber angle, the ciliary body was also infiltrated. Patients with tumors that infiltrated the anterior chamber angle had a mean BCVA of 0.94 (SD 0.50), versus 0.78 for patients without anterior chamber angle infiltration (SD 0.73, $p=0.50$ ). Similarly, two of two patients with tumor infiltration of the iris also had infiltration of the anterior chamber angle and ciliary body. These patients had a mean BCVA of 1.25 (SD 0.64 ), versus 0.77 for patients without tumor infiltration of the anterior chamber angle (SD $0.71, p=0.30$ ). Patients with partially necrotic tumors had a mean BCVA of 0.58 (SD 0.78), which was not significantly better than patients without tumor necrosis at 0.82 (SD $0.72, p=0.45$ ).

\section{Visual acuity versus symptoms}

Fifty-eight patients (85\%) reported a vision-related symptom (blurred vision/decreased VA, a shadow in the VF, photopsia and/or floaters, or metamorphopsia). One patient reported two symptoms. The mean BCVA for the group that reported at least one vision-related symptom was significantly better than the group that did not report any symptoms ( 0.73 , SD 0.70 and 1.3, SD 0.78 , respectively, Mann-Whitney $U$ $p=0.038)$.

Patients that experienced a shadow in the VF $(n=18)$ had significantly better BCVA than patients with no shadow $(0.38$, SD 0.51 and 0.95 , SD 0.73 , respectively, $p=0.002)$.

On the other hand, patients with PLVA $(n=33)$ did not have lower BCVA than patients without PLVA (0.90, SD 0.72 and 0.70, SD 0.72 , respectively, $p=0.15$ ). Similarly, there were no significant differences in BCVA between patients that reported metamorphopsia $(p=0.58)$ or photopsia and/or floaters $(p=0.67)$. No patients reported ocular pain, or any other symptom.

\section{Correlation matrix}

Patient sex, BCVA, PLVA, other symptoms, tumor size and location parameters and histological findings were entered into a correlation matrix. With Bonferroni correction, the significance level was reduced to 0.002. Positive relations of note included that a shadow in the VF correlated with presence of vasculogenic mimicry $(\rho 0.753, p<0.001)$; retinal detachment correlated with greater tumor distance to the macula $(\rho 0.814, p<0.001)$ and that presence of necrotic areas in the tumor correlated with heavy pigmentation $(\rho 0.419, \mathrm{p}<0.001)$. Conversely, PLVA correlated negatively with presence of vasculogenic mimicry in the tumor $(\rho-0.394, \mathrm{p}=0.001)$. Again, BCVA did not correlate with PLVA $(\rho 0.117, \mathrm{p}=0.34$, supplementary table).

\section{Survival}


Patients with tumors that infiltrated the optic nerve head had significantly shorter cumulative diseasespecific survival (Wilcoxon $p=0.024$, figure 3a). Similarly, patients with tumors that consisted of $>90 \%$ epithelioid tumor cells had worse prognosis than patients with a mix of epithelioid and spindle-like tumor cells, which in turn had worse prognosis than patients with $>90 \%$ spindle-like tumor cells (Wilcoxon $p$ for trend $<0.001$, figure $3 b$ ).

There were however no significant survival differences between patients with high and low BCVA $(p=0.50)$ or between presence, absence of any other histological finding or symptom. In a Cox regression model with tumor thickness and diameter entered as covariates, infiltration of the optic nerve head was associated with increased hazard for uveal melanoma-related mortality (HR 3.6, $95 \% \mathrm{Cl} 1.4$ to 9.2, $p=0.007$, figure 4a). Similarly, tumor cell type was associated with uveal melanoma-related mortality when adjusting for tumor thickness and diameter (HR 3.2, $95 \% \mathrm{Cl} 1.8$ to 5.6, $p<0.001$, figure 4b).

\section{Discussion}

In this paper, we have examined correlations between VA, patients' reported symptoms and histopathological findings in eyes with uveal melanoma. Quite unexpectedly, patients that experienced blurry vision or poor VA did not have significantly lower BCVA upon examination by an optometrist or ophthalmic nurse. The reason for this may be that patients with low BCVA or blindness are less likely to become aware of new symptoms or further changes to VA, especially if the changes are gradual and less pronounced. Patients with severely affected VA may also be more reliant on their healthy eye and less likely to report symptoms from the tumor eye.

We found no association between retinal detachment and poor BCVA. One reason for this may be that tumor-related retinal detachments are often peripheral and less likely to involve the central aspects of the retina. Even though detection of a faint peripheral shadow may be more dependent on the extent of an intact VF and the ability to discern luminance contrast, a certain degree of retained VA may be required to be able to perceive a shadow at all. A previous study by Fili et al. found that those who presented with a shadow in the VF had better BCVA, and tumors with grater thickness and larger diameters. ${ }^{13}$ This study found the same results regarding the better BCVA for patients who presented with a shadow in the VF.

Further, the experience of a shadow in the VF correlated with presence of vasculogenic mimicry in the tumor. The latter is a strong predictor of poor prognosis. ${ }^{26,27}$ The study by Fili et al. showed that the experience of a shadow in the VF was a consistent predictor of uveal melanoma-related mortality regardless of other simultaneous symptoms, tumor size, location, local extent, and stage. ${ }^{13}$ The reason that the experience of a shadow predicts poor prognosis may therefore be that these patients more often have tumors with vasculogenic mimicry. Based on the findings in these two studies, ocular oncologists may include the perception of a shadow in the visual field in the initial assessments of a patient's prognosis. Admittedly, it is not as strong a factor as gene expression class or analysis of BAP1 status and it is likely not suitable for a definite risk stratification. But it has the benefit of being rapidly 
assessable without examination and by just asking the patient simple question: "Do you perceive a shadow in your visual field?"

Other correlations of note include that low BCVA corresponds to larger tumors at a more advanced stage. This was not unexpected, considering that a larger tumor will affect a larger proportion of the retina. Patients with tumors that infiltrated the optic nerve head had significantly shorter cumulative diseasespecific survival and an increased hazard ratio for melanoma-related mortality even when adjusting for tumor diameter and thickness. This confirms the utility of optic nerve infiltration as a marker for poor prognosis, as previously reported by others. ${ }^{28,29}$

Limitations to this study include a relatively small retrospective sample without complete control over confounding factors. We had no access to data on loss of chromosome 3 heterozygosity, BAP1 mutations, BAP-1 expression or gene expression signatures, which are among the strongest prognostic factors in uveal melanoma. ${ }^{30,31}$ Further, our data did not include information about BCVA and the presence of disease in the contralateral eye. This may have affected patients' perceptions of symptoms and VA, and thereby our results herein. In several of the continuous variable group-level comparisons, the number of patients in specific groups was small, making results less generalizable. Only enucleated specimens were included, and the findings are not necessarily applicable to tumors undergoing plaque brachytherapy. However, we have no reasons to suspect that the examined correlations will be different in smaller tumors, including the role of presenting symptoms as previously published in a large cohort of patients treated with brachytherapy. ${ }^{13}$ Lastly, some of our measurements of histopathological characteristics were somewhat arbitrarily chosen due to lack of precedence. Factors not investigated here may be essential to VA in uveal melanoma.

\section{Conclusions}

Patients' experience of low VA does not necessarily correspond to low BCVA. The latter correlated with larger tumor dimensions and more advanced AJCC T category and stage. The experience of a shadow in the VF correlated with the presence of vasculogenic mimicry in the tumor, which is a marker for poor prognosis. Optic disc tumor infiltration was confirmed as a risk factor for uveal melanoma-related mortality, even when adjusting for tumor size.

\section{Methods}

The study follows the tenets of the Declaration of Helsinki and the research group's internal data security policy for personal data. Ethical permission was obtained from the Regional Ethics Review Board in Stockholm (record number 2016/347-31/4) with a supplement from the Swedish Ethical Review Authority (record number 2019-03485). According to the approved ethics application, the requirement for written informed consent was waived because this was a retrospective study that does not affect treatment or follow-up of the patients. Further, all patient data had been previously collected and no new clinical data 
collection was performed. No new tissues were collected, sectioned, stained or otherwise processed. The data was pseudonymized when histological sections were reviewed and then anonymized.

The cohort consisted of paraffin-embedded and formalin-fixed eyes that underwent primary enucleation for uveal melanoma between the years 2000 and 2007 at St. Erik Eye Hospital, Stockholm, Sweden. Inclusion criteria were: histologically proven melanoma originating from the choroid or ciliary body, sufficient formalin-fixed paraffin-embedded (FFPE) tissue for routine staining and correct representation of tumor histopathology, availability of clinical pathological data including primary tumor largest basal diameter and greatest tumor thickness. Exclusion criteria were: extensive tumor necrosis, hemorrhage or inflammation, heavily pigmented tumor affecting visual examination and suboptimal staining results, determined by positive and negative internal and external controls.

Seventy-five eyes met the inclusion criteria, of which seven were excluded ( $n=6$ no or too little tumor tissue represented in section, $n=1$ the represented tumor was completely necrotic). Sixty-eight eyes remained in the study. Retrospective information on gender, age at diagnosis, presenting symptoms, VA, symptom duration before presentation, tumor thickness (measured with A-and B-scan ultrasonography) and tumor diameter (measured in wide-field fundus photographs), American Joint Committee on Cancer (AJCC) T category, stage (supplementary figure) and date of diagnosis and final follow-up were obtained from our treatment registry. ${ }^{32}$ Patients had been asked to describe their symptoms and their duration in their own words. The ophthalmologist then recorded them as one or several of 1) blurred vision/decreased VA, 2) shadow in the VF, 3) photopsia and/or floaters, 4) metamorphopsia (confirmed with Amsler grid, if needed), 5) presence of ocular pain, or 6) other, including photophobia, diplopia and reduced perception of color.

Tumor sections were retrieved from the archives of the St. Erik Ocular Pathology Laboratory. For diagnostic purposes, these had previously been stained with hematoxylin and eosin, as well as periodic acid-Schiff (PAS) without hematoxylin counterstain. One representative section from each eye was digitally scanned at $\times 400$ using Nano Zoomer 2.0 HT scanner (Hamamatsu Photonics K.K., Hamamatsu, Japan) as described previously. ${ }^{33-35}$ The digitalization facilitated measurement of tumor dimension, distance to the macula, the diameter of necrotic areas and similar. The digitalized slides were examined by two authors (EA and GS).

\section{Visual acuity}

Best corrected visual acuity (BCVA): Each patient's VA was measured according to a standardized methodology one to three weeks prior to enucleation by an optometrist or ophthalmic nurse at the Ocular Oncology Service, St. Erik Eye Hospital. A KM-chart in an illuminated light box was used and patients tested at a distance of $3 \mathrm{~m} .{ }^{36}$ The BCVA recorded was the smallest line at which five of five or six of seven letters were correctly identified after subjective refraction and corrected in a trial frame. Patients could wear their own spectacles. All VA measurements reported refer to the tumor eye only (monocular) and were initially recorded on the decimal scale after which they were LogMAR-converted. 
Low and high BCVA: The measured BCVAs were dichotomized for this study so that high BCVA was defined as $\log M A R<1.00$ and low BCVA as $\log M A R \geq 1.00$, following a previously used classification. ${ }^{9}$

Perceived low VA (PLVA): If a patient described blurred vision, decreased VA, difficulties reading text or similar when prompted to describe his/her symptoms, and this was not deemed to represent a shadow in the VF, photopsia and/or floaters, metamorphopsia, photophobia, diplopia and reduced perception of color by the attending ophthalmologist, PLVA was recorded.

\section{Vasculogenic mimicry}

Patterns of microvascular loops and networks were assessed in a light microscope through a green narrow band pass filter according to the method described by Folberg et al. ${ }^{27}$ For statistical purposes, tumors were categorized into two groups based on the presence or absence of patterns with the strongest prognostic association: Extracellular networks, closed loops, arcs with branching, or any combination of these. ${ }^{27,37,38}$ This definition replicates one of our previous publications, in which these patterns correlated strongly to digitally measured density of Periodic acid-Schiff structures, loss of BAP-1 expression, gene expression class 2 and short metastasis-free survival. ${ }^{26}$ Further, the prognostic significance of the presence of loops, networks and combined patterns have been verified in several publications from other laboratories. ${ }^{24,39,40}$

\section{Statistical methods}

Differences with a $p<0.05$ were considered significant, all $p$-values being two-sided. Shapiro-Wilk tests were used to evaluate the deviation of continuous variables from normal distribution. If the test was significant $(p<0.05)$, the Mann Whitney U tests were used, otherwise Student's t-test were used. In comparisons of categorical variables, we used two-by-two contingency tables and Pearson chi-square $\left(x^{2}\right)$ tests (if all fields had a sample of $>5$ ) or Fisher's exact tests (if any field had a sample of $<5$ ). For comparisons of three groups or more, we used one-way ANOVA. LogMAR-converted VA levels were used throughout. VA levels less than $0.10,20 / 200$ or 1.00 (decimal scale, Snellen and LogMAR, respectively) were translated into numerical values according to standards from the Swedish National Quality Registry for Cataracts, in which counting fingers at a distance of $4 \mathrm{~m}$ is recorded as 0.08 on the decimal scale, hand movements as 0.04 , perception and localization of light as 0.01 and amaurosis as 0 . We built a correlation matrix of all histological findings, symptoms and VA and applied Spearman rank-order correlation statistics $(\rho)$. As this involved a large number of tests without preplanned hypotheses which will increase the risk for type I error, Bonferroni correction was applied. ${ }^{41}$ Lastly, the cumulative diseasespecific survival and Cox regression hazard ratios (HR) in relation to high and low BCVA, symptoms and histological findings were calculated. All statistical analyses were performed using IBM SPSS statistics version 27 (Armonk, NY).

\section{Declarations}

\section{Author contributions}


EA collected the data, contributed to data analysis and writing of the paper. TP, RB and MN contributed to data analysis and writing of the paper. GS conceived and designed the analysis, contributed to data analysis and writing of the paper. All authors read and approved the final manuscript.

\section{Competing interests}

The authors declare that they have no competing interests.

\section{Funding}

This work was supported by the following grants to Dr. Stålhammar:

- The Royal Swedish Academy of Sciences (reference ME2019-0036)

- The Swedish Cancer Society (20 0798 Fk)

- The Swedish Society of Medicine (Cronqvists stiftelse, reference SLS 934014)

- The Swedish Eye Foundation (reference 2020-04-27)

- Karolinska Institutet (reference 2020-013333 and 2020-02517)

- Region Stockholm (reference 20200356).

- The Crown Princess Margareta Foundation for the Visually Impaired (reference 157)

- Carmen and Bertil Regnér Foundation (reference 2020-00062)

\section{Availability of data and materials}

All data generated or analyzed during this study are included in this article and its supplementary material files. Further enquiries can be directed to the corresponding author.

\section{Ethics approval and consent to participate}

The study follows the tenets of the Declaration of Helsinki and the research group's internal data security policy for personal data. Ethical permission was obtained from the Regional Ethics Review Board in Stockholm (record number 2016/347-31/4) with a supplement from the Swedish Ethical Review Authority (record number 2019-03485). According to the approved ethics application, the requirement for written informed consent was waived because this was a retrospective study that does not affect treatment or follow-up of the patients. Further, all patient data had been previously collected and no new clinical data collection was performed. No new tissues were collected, sectioned, stained or otherwise processed. The data was pseudonymized when histological sections were reviewed and then anonymized.

\section{Consent to participate}

Not applicable 


\section{Consent for publication}

Not applicable

\section{References}

1. Kaliki, S., Shields, C. L. \& Shields, J. A. Uveal melanoma: estimating prognosis. Indian J Ophthalmol 63, 93-102, doi:10.4103/0301-4738.154367 (2015).

2. Shields, C. L. et al. Metastasis of Uveal Melanoma Millimeter-by-Millimeter in 8033 Consecutive Eyes. Archives of Ophthalmology 127, 989-998, doi:10.1001/archophthalmol.2009.208 (2009).

3. Kujala, E., Mäkitie, T. \& Kivelä, T. Very long-term prognosis of patients with malignant uveal melanoma. Invest Ophthalmol Vis Sci 44, 4651-4659 (2003).

4. Stalhammar, G. Forty-year prognosis after plaque brachytherapy of uveal melanoma. Sci Rep 10, 11297, doi:10.1038/s41598-020-68232-7 (2020).

5. COMS. The COMS Randomized Trial of lodine 125 Brachytherapy for Choroidal Melanoma: V. Twelve-Year Mortality Rates and Prognostic Factors: COMS Report No. 28. Archives of Ophthalmology 124, 1684-1693, doi:10.1001/archopht.124.12.1684 (2006).

6. Melia, B. M. et al. Collaborative ocular melanoma study (COMS) randomized trial of I-125 brachytherapy for medium choroidal melanoma. I. Visual acuity after 3 years COMS report no. 16 . Ophthalmology 108, 348-366, doi:10.1016/s0161-6420(00)00526-1 (2001).

7. Tamplin, M. R. et al. Temporal Relationship Between Visual Field, Retinal and Microvascular Pathology Following 125I-Plaque Brachytherapy for Uveal Melanoma. Invest Ophthalmol Vis Sci 62, 3, doi:10.1167/iovs.62.1.3 (2021).

8. Wisely, C. E. et al. Long-term visual acuity outcomes in patients with uveal melanoma treated with 125I episcleral OSU-Nag plaque brachytherapy. Brachytherapy 15, 12-22, doi:10.1016/j.brachy.2015.09.013 (2016).

9. Shields, C. L. et al. Plaque radiotherapy for uveal melanoma: long-term visual outcome in 1106 consecutive patients. Arch Ophthalmo/ 118, 1219-1228, doi:10.1001/archopht.118.9.1219 (2000).

10. Bergman, L., Nilsson, B., Lundell, G., Lundell, M. \& Seregard, S. Ruthenium brachytherapy for uveal melanoma, 1979-2003: survival and functional outcomes in the Swedish population. Ophthalmology 112, 834-840, doi:10.1016/j.ophtha.2004.11.038 (2005).

11. Dalvin, L. A. et al. Nomogram for visual acuity outcome after iodine-125 plaque radiotherapy and prophylactic intravitreal bevacizumab for uveal melanoma in 1131 patients. $\mathrm{Br} \mathrm{J} \mathrm{Ophthalmo/} \mathrm{104,}$ 697-702, doi:10.1136/bjophthalmol-2019-314686 (2020).

12. Damato, E. M. \& Damato, B. E. Detection and time to treatment of uveal melanoma in the United Kingdom: an evaluation of 2,384 patients. Ophthalmology 119, 1582-1589, doi:10.1016/j.ophtha.2012.01.048 (2012). 
13. Fili, M., Seregard, S. \& Stalhammar, G. Presenting Symptoms Are Associated with Uveal MelanomaRelated Death. Ophthalmology, doi:10.1016/j.ophtha.2020.11.023 (2020).

14. COMS. Histopathologic characteristics of uveal melanomas in eyes enucleated from the Collaborative Ocular Melanoma Study. COMS report no. 6. Am J Ophthalmo/ 125, 745-766, doi:10.1016/s0002-9394(98)00040-3 (1998).

15. Schoenfield, L. Uveal melanoma: A pathologist's perspective and review of translational developments. Adv Anat Patho/ 21, 138-143, doi:10.1097/PAP.0000000000000010 (2014).

16. Saornil, M. A. et al. Histopathology of proton beam-irradiated vs enucleated uveal melanomas. Arch Ophthalmol 110, 1112-1118, doi:10.1001/archopht.1992.01080200092031 (1992).

17. McLean, I. W., Foster, W. D., Zimmerman, L. E. \& Gamel, J. W. Modifications of Callender's classification of uveal melanoma at the Armed Forces Institute of Pathology. Am J Ophthalmo/ 96, 502-509, doi:10.1016/s0002-9394(14)77914-0 (1983).

18. Callender, G., Campbell Wilder, H. \& Ash, J. Five Hundred Melanomas of the Choroid and Ciliary Body Followed Five Years Or Longer. American Journal of Ophthalmology 25, 962-967, doi:https://doi.org/10.1016/S0002-9394(42)90595-6 (1942).

19. Callender, G. Malignant melanotic tumors of the eye: a study of histologic types in 111 cases. Trans Am Acad Ophthalmol Otolaryngol 36, 131-142 (1931).

20. Gamel, J. W., McLean, I. W., Foster, W. D. \& Zimmerman, L. E. Uveal melanomas: correlation of cytologic features with prognosis. Cancer 41, 1897-1901, doi:10.1002/10970142(197805)41:5<1897::aid-cncr2820410534>3.0.co;2-2 (1978).

21. Zimmerman, L. E. Pathology and computed tomography. Ophthalmology 87, 602-605, doi:10.1016/s0161-6420(80)35211-1 (1980).

22. McLean, I. W., Foster, W. D. \& Zimmerman, L. E. Uveal melanoma: location, size, cell type, and enucleation as risk factors in metastasis. Hum Pathol 13, 123-132, doi:10.1016/s00468177(82)80116-0 (1982).

23. Folberg, R. et al. Mapping the Location of Prognostically Significant Microcirculatory Patterns in Ciliary Body and Choroidal Melanomas. Pathol Oncol Res 2, 229-236 (1996).

24. McLean, I. W., Keefe, K. S. \& Burnier, M. N. Uveal melanoma. Comparison of the prognostic value of fibrovascular loops, mean of the ten largest nucleoli, cell type, and tumor size. Ophthalmology 104, 777-780, doi:10.1016/S0161-6420(97)30234-6 (1997).

25. Herrspiegel, C. et al. Nuclear expression of BAP-1 in transvitreal incisional biopsies and subsequent enucleation of eyes with posterior choroidal melanoma. Br J Ophthalmol 105, 582-586, doi:10.1136/bjophthalmol-2020-316498 (2021).

26. Stålhammar, G., See, T. R. O., Phillips, S. S. \& Grossniklaus, H. E. Density of PAS positive patterns in uveal melanoma: Correlation with vasculogenic mimicry, gene expression class, BAP-1 expression, macrophage infiltration, and risk for metastasis. Molecular Vision 25, 502-516 (2019).

27. Folberg, R. et al. The morphologic characteristics of tumor blood vessels as a marker of tumor progression in primary human uveal melanoma: A matched case-control study. Human Pathology 
23, 1298-1305, doi:10.1016/0046-8177(92)90299-I (1992).

28. Weinhaus, R. S., Seddon, J. M., Albert, D. M., Gragoudas, E. S. \& Robinson, N. Prognostic factor study of survival after enucleation for juxtapapillary melanomas. Arch Ophthalmo/ 103, 1673-1677, doi:10.1001/archopht.1985.01050110067027 (1985).

29. Lindegaard, J., Isager, P., Prause, J. U. \& Heegaard, S. Optic nerve invasion of uveal melanoma: clinical characteristics and metastatic pattern. Invest Ophthalmol Vis Sci 47, 3268-3275, doi:10.1167/iovs.05-1435 (2006).

30. Harbour, J. W. et al. Frequent mutation of BAP1 in metastasizing uveal melanomas. Science 330, 1410-1413, doi:10.1126/science.1194472 (2010).

31. Prescher, G. et al. Prognostic implications of monosomy 3 in uveal melanoma. Lancet 347, 12221225, doi:10.1016/s0140-6736(96)90736-9 (1996).

32. Kivelä, T. et al. in AJCC Cancer Staging Manual Ch. 67, 805-817 (Springer, 2017).

33. Herrspiegel, C., See, T. R. O., Mendoza, P. R., Grossniklaus, H. E. \& Stålhammar, G. Digital morphometry of tumor nuclei correlates to BAP-1 status, monosomy 3, gene expression class and survival in uveal melanoma. Exp Eye Res 193, 107987, doi:10.1016/j.exer.2020.107987 (2020).

34. See, T. R. O., Stålhammar, G., Phillips, S. S. \& Grossniklaus, H. E. BAP1 Immunoreactivity Correlates with Gene Expression Class in Uveal Melanoma. Ocular Oncology and Pathology, 1-9, doi:DOI:10.1159/000502550 (2019).

35. Stålhammar, G., See, T. R. O., Phillips, S., Seregard, S. \& Grossniklaus, H. E. Digital Image Analysis of BAP-1 Accurately Predicts Uveal Melanoma Metastasis. Translational vision science \& technology $\mathbf{8}$, 11, doi:10.1167/tvst.8.3.11 (2019).

36. Moutakis, K., Stigmar, G. \& Hall-Lindberg, J. Using the KM visual acuity chart for more reliable evaluation of amblyopia compared to the HVOT method. Acta Ophthalmol Scand 82, 547-551, doi:10.1111/j.1600-0420.2004.00307.x (2004).

37. Folberg, R. et al. The prognostic value of tumor blood vessel morphology in primary uveal melanoma. Ophthalmology 100, 1389-1398, doi:10.1016/s0161-6420(93)31470-3 (1993).

38. Rummelt, V., Folberg, R., Woolson, R. F., Hwang, T. \& Pe'er, J. Relation between the microcirculation architecture and the aggressive behavior of ciliary body melanomas. Ophthalmology 102, 844-851, doi:10.1016/s0161-6420(95)30947-5 (1995).

39. Foss, A. J. E. et al. Reassessment of the PAS patterns in uveal melanoma. British Journal of Ophthalmology 81, 240, doi:10.1136/bjo.81.3.240 (1997).

40. Makitie, T., Summanen, P., Tarkkanen, A. \& Kivela, T. Microvascular loops and networks as prognostic indicators in choroidal and ciliary body melanomas. J Natl Cancer Inst 91, 359-367, doi:10.1093/jnci/91.4.359 (1999).

41. Armstrong, R. A. When to use the Bonferroni correction. Ophthalmic Physiol Opt 34, 502-508, doi:10.1111/opo.12131 (2014). 
Figures

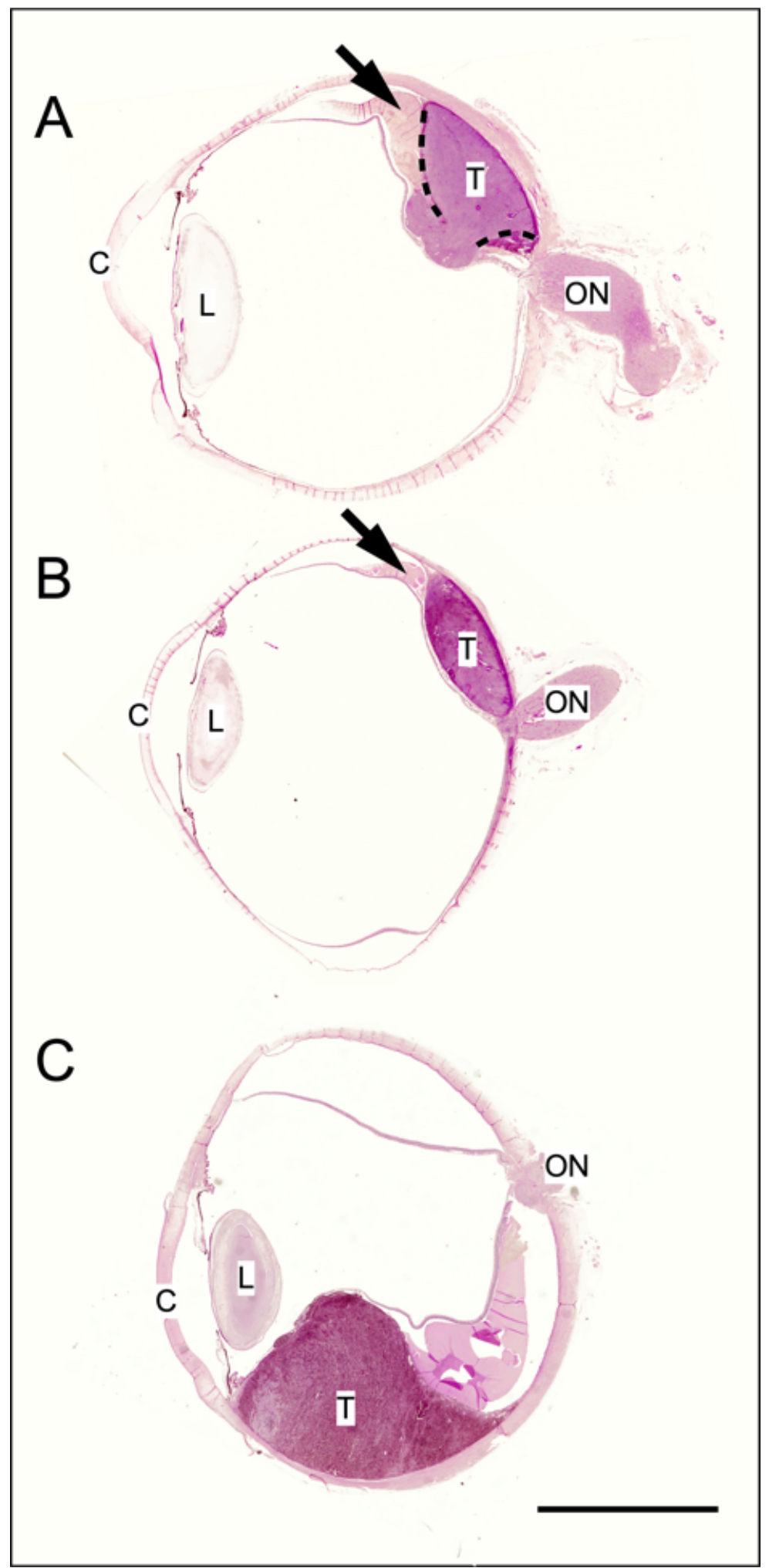

\section{Figure 1}

Examples of choroidal and ciliary body melanomas. A) A tumor in close proximity to but not touching the optic disc. The mushroom or collar-button shape is a result of focal tumor penetration of Bruch's membrane. The location of Bruch's membrane is indicated (dashed lines). A serous exudate is present 
under the detached retina (arrow). Remnants of an enlarged lens can be seen. B) A small dome-shaped tumor touching the optic disc. This tumor is away from the central axis of the eye and may affect visual acuity to a lower degree than a tumor under the macula. A small serous exudate is present near the anterior tumor base (arrow). C) A large ciliary body melanoma with significant infiltration in the choroid, heavier pigmentation, and exudative retinal detachment. C, Cornea. L, Lens. T, Tumor. ON, Optic nerve. Scale bar: $10 \mathrm{~mm}$.
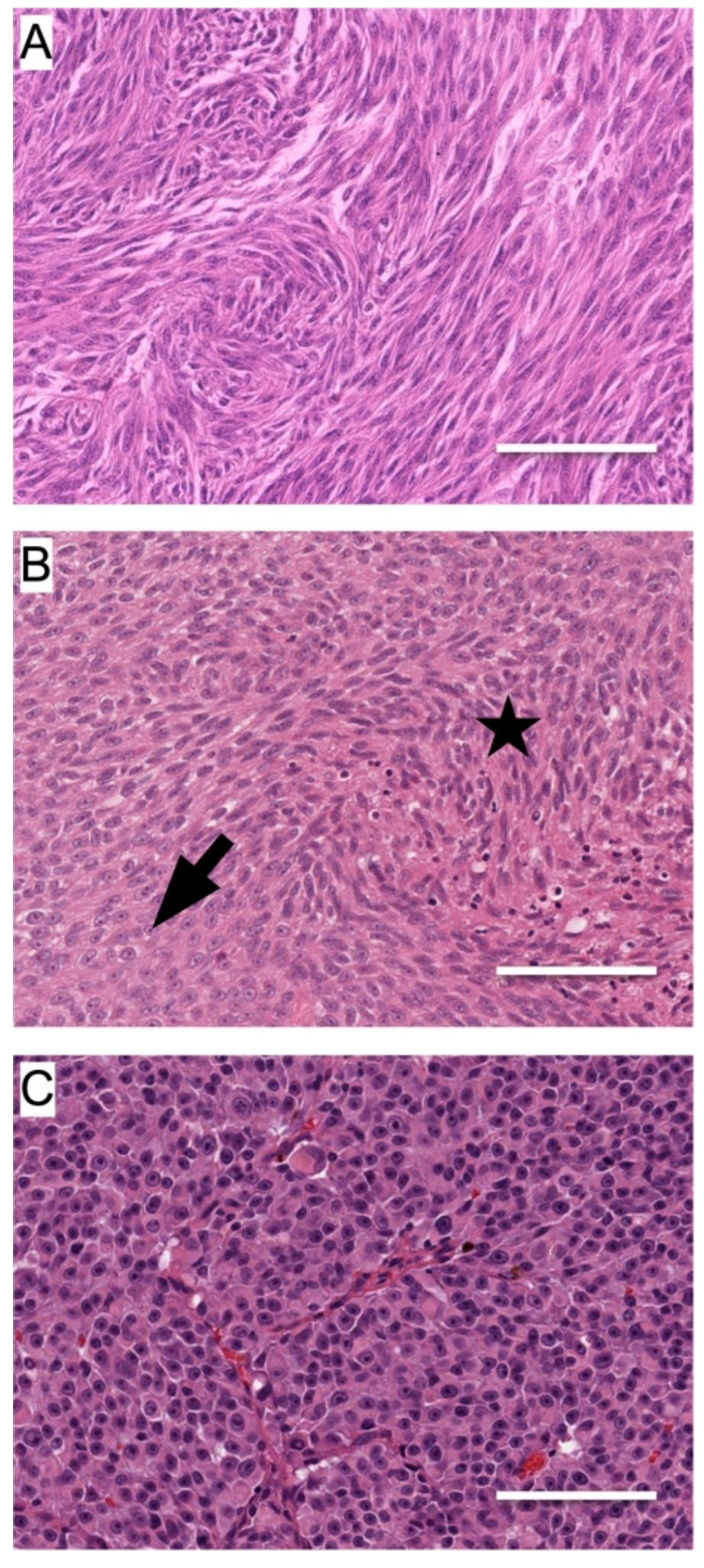

Figure 2 
Examples of tumor cell types. A) A spindle cell tumors consist of $>90 \%$ spindle cells. In turn, spindle cells may be further subclassified as spindle cell type A and spindle cell type B. Spindle A cells have long, tapering cigar-like nuclei, an absent or indistinct nucleolus, and a characteristic longitudinal stripe caused by a fold in the nuclear membrane. Spindle B nuclei are oval and plumper and have less finely dispersed chromatin and a distinct nucleolus. In this study, no distinction was made between different types of spindle cells. B) Mixed tumors consist of cells with both epithelioid (arrow) and spindle-like shape (star). C) Epithelioid tumors consist of $>90 \%$ epithelioid cells with round, plump nuclei and distinct nucleoli. Scale bars $100 \mu \mathrm{m}$. 

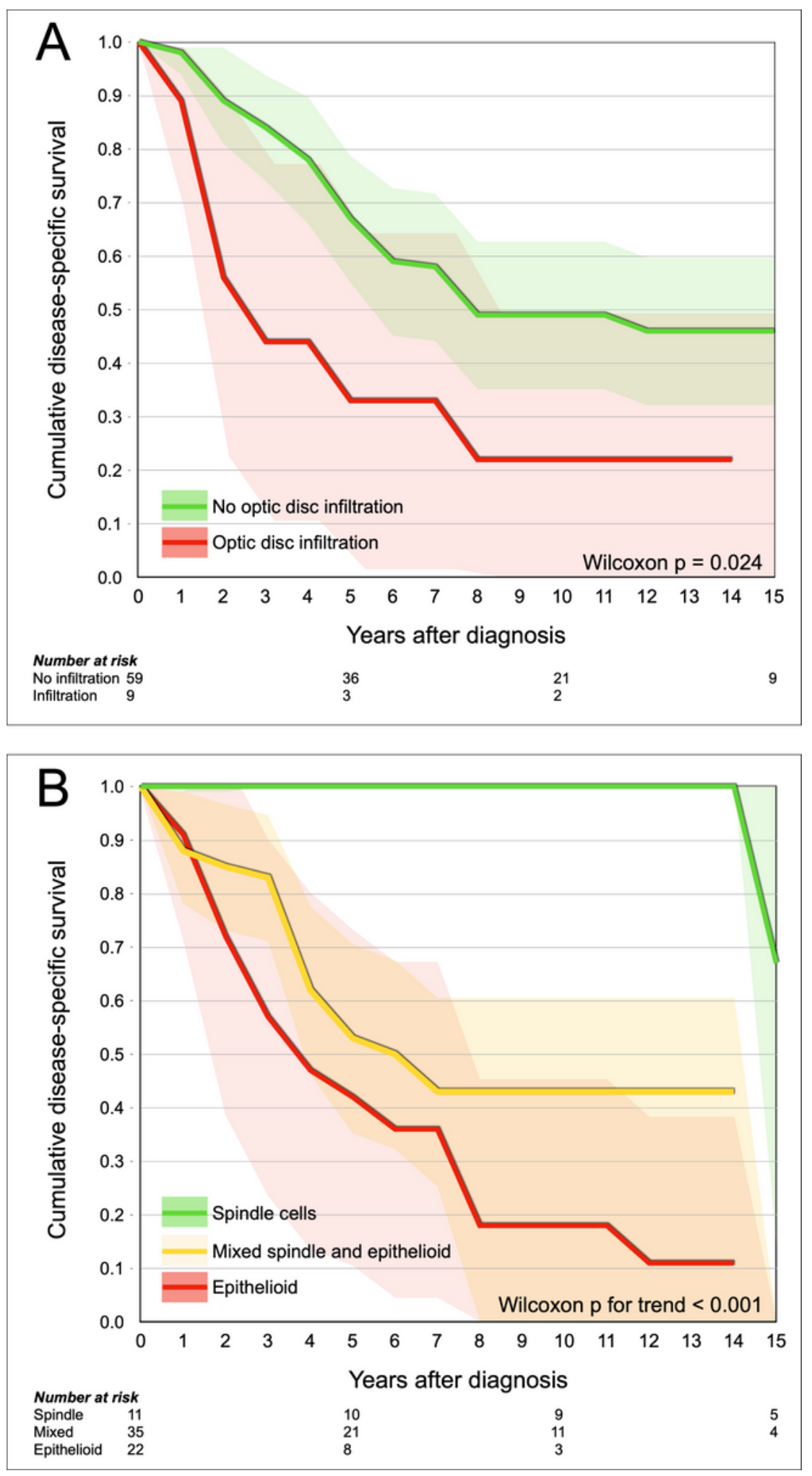

Figure 3

Survival analysis. A) Patients with prelaminar optic disc tumor infiltration (red) had significantly shorter cumulative disease-specific survival than patients without disc infiltration (green, Wilcoxon $p=0.024$ ). B) Patients with tumors that consisted of $>90 \%$ epithelioid cells (red) had the shortest cumulative diseasespecific survival, followed by mixed cells (yellow) and $>90 \%$ spindle-like cells (green, Wilcoxon $p$ for trend<0.001). Colored areas represent $95 \% \mathrm{Cl}$. 

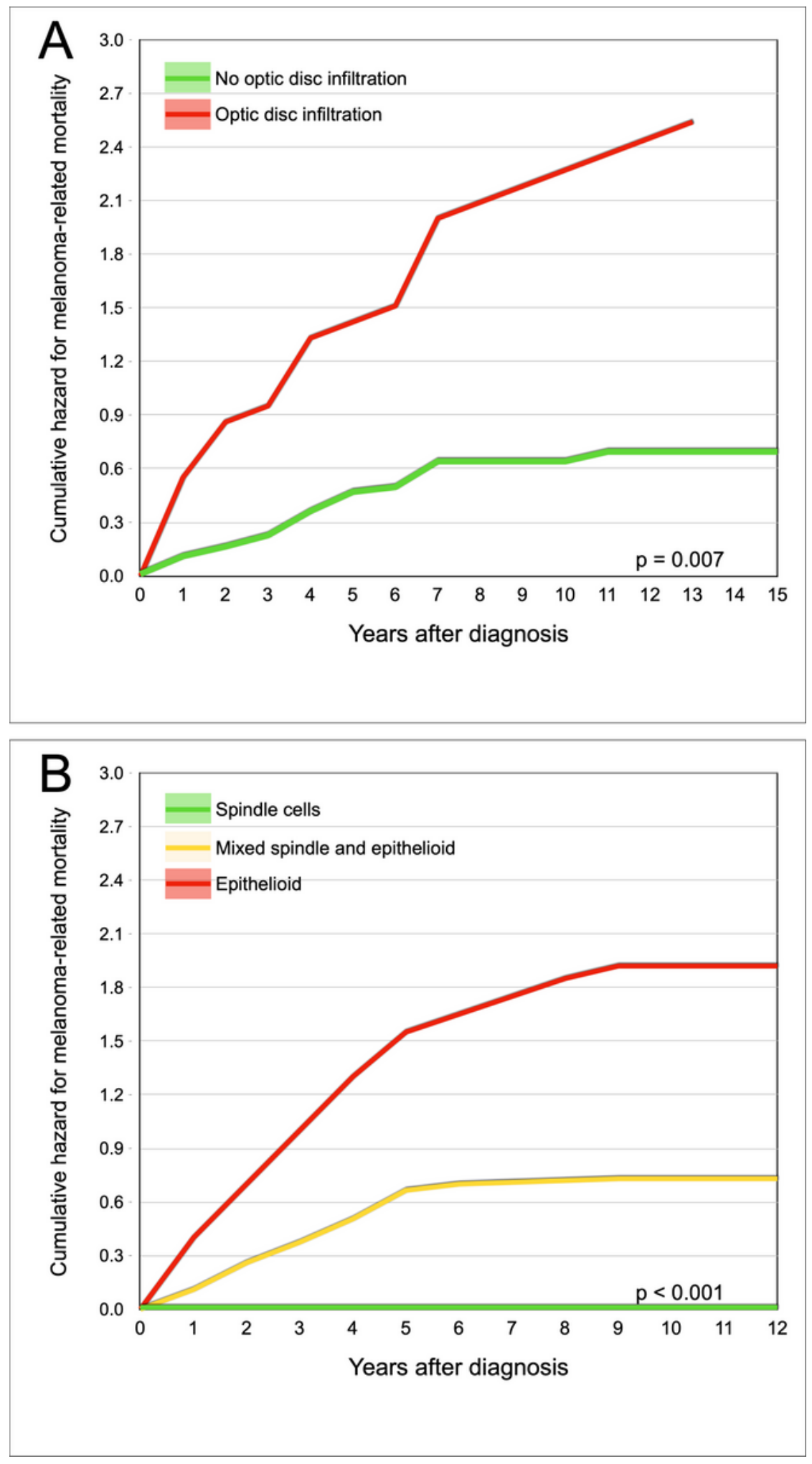

\section{Figure 4}

Multivariate Cox Regression analyses. A) Patients with prelaminar optic disc tumor infiltration (red) had significantly greater cumulative Cox Regression hazard for melanoma-related mortality than patients without optic disc infiltration (green), adjusted for tumor thickness and diameter (HR 3.6, $95 \% \mathrm{Cl} 1.4$ to $9.2, p=0.007)$. B) Similarly, patients with tumors that consisted of $>90 \%$ epithelioid cells (red) had significantly greater cumulative Cox Regression hazard for melanoma-related mortality than patients with 
mixed cells (yellow) and >90\% spindle-like cells (green), adjusted for tumor thickness and diameter (HR $3.2,95 \% \mathrm{Cl} 1.8$ to $5.6, \mathrm{p}<0.001)$.

\section{Supplementary Files}

This is a list of supplementary files associated with this preprint. Click to download.

- Supplementaryfigure.pdf

- Supplementarytable.pdf 